\title{
Inequalities on Hardy and higher-power weighted Bergman spaces of composition operators
}

\author{
Elhadi Elniel $^{\mathrm{a}, *}$, Shawgy Hussein ${ }^{\mathrm{b}}$ \\ a Department of Mathematics, College of Science and Arts, Al-Baha University, Saudi Arabia \\ b Department of Mathematics, College of Science, Sudan University of Science and Technology, Sudan
}

*Corresponding author, e-mail: elhadielniel_2003@hotmail.com

Received 13 Feb 2014

Accepted 9 Jun 2014

\begin{abstract}
Bounded composition operators are usually induced by analytic self-maps of the open unit disk acting on the Hardy space $H^{2}$ and on the higher-power weighted Bergman spaces $L_{e_{\alpha}}^{2}$ where $e_{\alpha}=(\alpha+1)^{2}-1$. An inequality for the relationship between the norms of the corresponding composition operators defined on these spaces is considered.
\end{abstract}

KEYWORDS: norm inequalities, semidefinite matrices, Schur product theorem

\section{INTRODUCTION}

Let $D$ be the open unit disk in the complex plane and let $\varphi: D \rightarrow D$ be an analytic self-map. If $\mathcal{H}$ is a Hilbert space of analytic functions $f: D \rightarrow \mathbb{C}$, the composition operator $C_{\varphi}$ on $\mathcal{H}$ is defined by $C_{\varphi}(f)=$ $f \circ \varphi$ for all $f \in \mathcal{H}$. While there are some Hilbert spaces (for example, the Dirichlet space) where the composition operators are unbounded, every analytic $\varphi$ induces a bounded operator on all of the spaces considered in this paper. We show relationships between the operator norms of $C_{\varphi}$ acting on different spaces with weights.

The Hilbert spaces of primary interest to us will be the Hardy space $H^{2}$ and the power weighted Bergman spaces $L_{e_{\alpha}}^{2}$ where $e_{\alpha}=(\alpha+1)^{2}-1$. The Hardy space consists of all analytic functions $f$ on $D$ such that

$$
\|f\|_{H^{2}}^{2}=\frac{1}{2 \pi} \sup _{0<r<1} \int_{0}^{2 \pi}\left|f\left(r \mathrm{e}^{i \theta}\right)\right|^{2} d \theta<\infty
$$

with the inner product

$$
\langle f, g\rangle_{H^{2}}=\frac{1}{2 \pi} \lim _{r \rightarrow 1^{-}} \int_{0}^{2 \pi} f\left(r \mathrm{e}^{i \theta}\right) \overline{g\left(r \mathrm{e}^{i \theta}\right)} d \theta<\infty .
$$

The Hardy space can be described as a reproducing kernel Hilbert space, since for every point $\lambda \in D$ there is a unique function $K_{\lambda} \in H^{2}$ such that $\left\langle f, K_{\lambda}\right\rangle_{H^{2}}=$ $f(\lambda)$ for all $f \in H^{2}$; in fact, $K_{\lambda}(z)=1 /(1-\bar{\lambda} z)$ (see Ref. 1).

For $\alpha>-1$, we define the power weighted Bergman space, denoted $L_{e_{\alpha}}^{2}$, to be the space of all analytic functions $f$ on $D$ such that

$$
\|f\|_{L_{e_{\alpha}}^{2}}^{2}=\int_{D}|f(z)|^{2}(\alpha+1)^{2}\left(1-|z|^{2}\right)^{e_{\alpha}} \mathrm{d} A<\infty,
$$

where $\mathrm{d} A$ is the normalized area measure on $D$.

We write $\langle\cdot, \cdot\rangle_{L_{e_{\alpha}}^{2}}$, for any $\alpha$, to denote the inner product on $L_{e_{\alpha}}^{2}$ with the kernel function $k_{\lambda}^{e_{\alpha}}(z)=$ $1 /(1-\bar{\lambda} z)^{e_{\alpha}+2}$. There is an obvious likeness between the reproducing kernels for $H^{2}$ and the analogous functions for $L_{e_{\alpha}}^{2}$. For the sake of efficiency, we write $L_{-1}^{2}$ to denote the Hardy space $H^{2}$, with $k_{\lambda}^{-1}=K_{\lambda}$ and $\langle\cdot, \cdot\rangle_{L_{-1}^{2}}=\langle\cdot, \cdot\rangle_{H^{2}}$. We will state many of the results in these terms, with the understanding that the $\alpha=0$ and $\alpha=-2$ power weighted Bergman spaces always signifies the Hardy space.

For any analytic $\varphi: D \rightarrow D$, we will write $\left\|C_{\varphi}\right\|_{\mathcal{H}}$ to denote the norm of $C_{\varphi}$ acting on a Hilbert space $\mathcal{H}$. While, it is generally not easy to calculate the norm $\left\|C_{\varphi}\right\|_{L_{e_{\alpha}}^{2}}$ explicitly $^{2-5}$, it is in fact not difficult to estimate the norm of $C_{\varphi}$. In particular, it is well known that

$$
\begin{aligned}
\left(\frac{1}{1-|\varphi(0)|^{2}}\right)^{e_{\alpha}+2} & \leqslant\left\|C_{\varphi}\right\|_{L_{e_{\alpha}}^{2}}^{2} \\
& \leqslant\left(\frac{1+|\varphi(0)|}{1-|\varphi(0)|^{2}}\right)^{e_{\alpha}+2}
\end{aligned}
$$

for any $\alpha \geqslant-1$ (see Refs. 1,6). In spite of (1), one might wonder whether there is some relationship between the quantities $\left\|C_{\varphi}\right\|_{L_{e_{\alpha}}^{2}}$ for different values of $\alpha$. 
For example, considering $\alpha=0, \alpha=-1$ and $\alpha=-2$, one might ask whether it is always the case that $\left\|C_{\varphi}\right\|_{L^{2}}=\left\|C_{\varphi}\right\|_{H^{2}}^{2}$. While this equality does hold for some maps, it is not true in general ${ }^{7}$. Christopher Hammond and Linda J. Patton ${ }^{8}$ proved that $\left\|C_{\varphi}\right\|_{L^{2}} \leqslant\left\|C_{\varphi}\right\|_{H^{2}}^{2}$ for all $\varphi$ answering a question posed by Carswell and Hammond ${ }^{7}$, and they derived a collection of inequalities relating to the norms of $C_{\varphi}$ acting on different spaces.

In this paper we apply norm inequalities for composition operators ${ }^{8}$ to give a verification of higherpower weighted Bergman spaces. Now we should mention a helpful fact relating to composition operators and reproducing kernel functions. Let $C_{\varphi}^{*}$ denote the adjoint of $C_{\varphi}$ on a particular space $L_{e_{\alpha}}^{2}$, and that $C_{\varphi}^{*}\left(K_{\lambda}^{e_{\alpha}}\right)=K_{\varphi(\lambda)}^{e_{\alpha}}$ for any $\lambda \in D$ (see Ref. 1). This observation will provide exactly the verification of the information we need to compare the action of $C_{\varphi}$ on different spaces.

\section{POSITIVE SEMIDEFINITE MATRICES}

Let $\Lambda=\left\{\lambda_{m}\right\}_{m=1}^{\infty}$, a sequence of distinct points in $D$, be a set of uniqueness for the collection of analytic functions on $D$. In other words, the zero function is the only analytic function with $f\left(\lambda_{m}\right)=0$ for all $m$. The span of the kernel functions $\left\{k_{\lambda_{m}}^{e_{\alpha}}\right\}_{m=1}^{\infty}$ is dense in every space $L_{e_{\alpha}}^{2}$, since any function orthogonal to every $k_{\lambda_{m}}^{e_{\alpha}}$ must be identically 0 . Throughout this paper, we will assume that such a sequence $\Lambda$ has been fixed.

Consider an analytic map $\varphi: D \rightarrow D$. For a positive constant $\nu$, a natural number $n$, and a real number $\alpha \geqslant-1$, we define the $n \times n$ matrix $M(\nu, n, \alpha(\alpha+2))=\left(m_{i j}\right)_{n \times n}$ by

$$
m_{i j}=\frac{\nu^{2}}{\left(1-\bar{\lambda}_{j} \lambda_{i}\right)^{e_{\alpha}+2}}-\frac{1}{\left(1-\overline{\varphi\left(\lambda_{j}\right)} \varphi\left(\lambda_{i}\right)\right)^{e_{\alpha}+2}}
$$

where $e_{\alpha}=(\alpha+1)^{2}-1$. In particular, we put

$$
M=\operatorname{diag}\left(\frac{\nu^{2}}{\left(1-|\lambda|^{2}\right)^{e_{\alpha}+2}}-\frac{1}{\left(1-\left|\varphi\left(\lambda_{j}\right)\right|^{2}\right)^{e_{\alpha}+2}}\right) .
$$

Recall that an $n \times n$ matrix $A$ is called positive semidefinite if $\langle A c, c\rangle \geqslant 0$ for all $c \in \mathbb{C}^{n}$, denoted $A \geqslant 0$ where $\langle\cdot, \cdot\rangle$ is the standard Euclidean inner product. Any such matrix must necessarily be self-adjoint. For self-adjoint matrices $A$ and $B$, we write $A \geqslant B$ if $A$ $B \geqslant 0$. The following proposition relates $\left\|C_{\varphi}\right\|_{L_{e_{\alpha}}^{2}}$ to the positive semi-definiteness of $M(\nu, n, \alpha(\alpha+2))$.

Proposition 1 Let $\varphi: D \rightarrow D$ be an analytic selfmap and $n$ be a positive constant. Then, for any $\alpha \geqslant-1$, the matrix $M(\nu, n, \alpha(\alpha+2))$ is positive semi-definite for all natural numbers $n$ if and only if $\left\|C_{\varphi}\right\|_{L_{e_{\alpha}}^{2}} \leqslant \nu$.

Proof: Assume first that $\left\|C_{\varphi}\right\|_{L_{e_{\alpha}}^{2}} \leqslant \nu$, from which it follows that $\left\|C_{\varphi}^{*}\right\|_{L_{e_{\alpha}}^{2}} \leqslant \nu$. In other words, we have

$$
\left\|C_{\varphi}^{*}(f)\right\|_{L_{e_{\alpha}}^{2}}^{2} \leqslant \nu^{2}\|f\|_{L_{e_{\alpha}}^{2}}^{2} .
$$

Let $f \in L_{e_{\alpha}}^{2}$ and $c_{1}, \ldots, c_{n} \in \mathbb{C}$. We express $f=$ $\sum_{j=1}^{n} c_{j} k_{\lambda_{j}}^{e_{\alpha}}$. If we substitute this function into (2), recalling that $C_{\varphi}^{*}\left(k_{\lambda}^{e_{\alpha}}\right)=k_{\varphi(\lambda)}^{e_{\alpha}}$, then we obtain

$$
\left\|\sum_{j=1}^{n} c_{j} k_{\varphi\left(\lambda_{j}\right)}^{e_{\alpha}}\right\|_{L_{e_{\alpha}}^{2}}^{2} \leqslant \nu^{2}\left\|\sum_{j=1}^{n} c_{j} k_{\lambda_{j}}^{e_{\alpha}}\right\|_{L_{e_{\alpha}}^{2}}^{2}
$$

from which it follows that

$$
\sum_{j=1}^{n}\left|c_{j}\right|^{2}\left\|k_{\varphi\left(\lambda_{j}\right)}^{e_{\alpha}}\right\|_{L_{e_{\alpha}}^{2}}^{2} \leqslant \sum_{j=1}^{n} \nu^{2}\left|c_{j}\right|^{2}\left\|k_{\lambda_{j}}^{e_{\alpha}}\right\|_{L_{e_{\alpha}}^{2}}^{2}
$$

and thus

$$
\begin{aligned}
\sum_{j=1}^{n}\left|c_{j}\right|^{2}\left[\frac{\nu^{2}}{\left(1-\left|\lambda_{j}\right|^{2}\right)^{e_{\alpha}+2}}\right. & \\
& \left.-\frac{1}{\left(1-\left|\varphi\left(\lambda_{j}\right)\right|^{2}\right)^{e_{\alpha}+2}}\right] \geqslant 0 .
\end{aligned}
$$

Inequality (3) precisely implies that $M(\nu, n, \alpha(\alpha+2))$ is positive semi-definite.

For the converse, assume that $M(\nu, n, \alpha(\alpha+2))$ is positive semi-definite for all natural numbers $n$. Hence (3) holds for all $n$, which in turn implies that

$$
\left\|\sum_{j=1}^{n} c_{j} k_{\varphi\left(\lambda_{j}\right)}^{e_{\alpha}}\right\|_{L_{e_{\alpha}}^{2}}^{2} \leqslant \nu^{2}\left\|\sum_{j=1}^{n} c_{j} k_{\lambda_{j}}^{e_{\alpha}}\right\|_{L_{e_{\alpha}}^{2}}^{2} .
$$

For any $n$ and any complex numbers $c_{1}, \ldots, c_{n}$, let $f$ be an arbitrary element of $L_{e_{\alpha}}^{2}$. Since $\Lambda$ is a set of uniqueness, the span of $\left\{k_{\lambda_{n}}^{\left.e_{\alpha}\right\}_{n=1}^{\infty}}\right.$ is dense in $L_{e_{\alpha}}^{2}$. Hence there exists a sequence $\left\{f_{m}\right\}_{m=1}^{\infty}$ that converges to $f$ in norm, where each $f_{m}$ is a finite linear combination of these kernel functions. The inequality of (4) implies that $\left\|C_{\varphi}^{*}\left(f_{m}\right)\right\|_{L_{e_{\alpha}}^{2}}^{2} \leqslant \nu^{2}\left\|f_{m}\right\|_{L_{e_{\alpha}}^{2}}^{2}$ for all $m$.

Letting $m \rightarrow \infty$, we see that $\left\|C_{\varphi}^{*}(f)\right\|_{L_{e_{\alpha}}^{2}}^{2} \leqslant$ $\nu^{2}\|f\|_{L_{e_{\alpha}}^{2}}^{2}$, from which it follows (upon taking the supremum over all $f \in L_{e_{\alpha}}^{2}$ ) that

$$
\left\|C_{\varphi}\right\|_{L_{e_{\alpha}}^{2}}=\left\|C_{\varphi}^{*}\right\|_{L_{e_{\alpha}}^{2}} \leqslant \nu
$$


Hence Proposition 1 states that $\left\|C_{\varphi}\right\|_{L_{e_{\alpha}}^{2}} \leqslant \nu$ exactly when

$$
k_{\lambda}(z)=\frac{\nu^{2}}{(1-\bar{\lambda} z)^{e_{\alpha}+2}}-\frac{1}{(1-\overline{\varphi(\lambda)} \varphi(z))^{e_{\alpha}+2}}
$$

is a positive semi definite kernel on the unit disk.

Remark 1 If $f^{r}=\sum_{j=1}^{n} c_{j} k_{\lambda_{j}}^{\left(\alpha_{r}+1\right)^{2}-1}$ where $r=$ $1,2, \ldots, n$. Proposition 1 implies that $f_{m}^{r} \rightarrow f^{r}$ uniformly in the norm. We can deduce that

$$
\left\|c^{*}\left(\sum_{r=1}^{n} f^{r}\right)\right\|_{L_{\left(\alpha_{r}+1\right)^{2}-1}^{2}}^{2} \leqslant \nu^{2} \sum_{r=1}^{n}\left\|f^{r}\right\|_{L_{\left(\alpha_{r}+1\right)^{2}-1}^{2}}^{2} .
$$

We need the following lemma which relating to positive semi-definite matrices.

Lemma 1 Let $\lambda_{1}, \ldots, \lambda_{n}$ be a finite collection of (not necessarily distinct) points in $D$. Any matrix of the form

$$
M=\left[\frac{1}{\left(1-\overline{\lambda_{j}} \lambda_{i}\right)^{\rho}}\right]_{i, j=1}^{n},
$$

for any real number $\rho \geqslant 1$, must be positive semidefinite, and so is a diagonal matrix

$$
\operatorname{diag}\left(\frac{1}{\left(1-\left|\lambda_{j}\right|^{2}\right)^{\rho}}\right)_{j=1}^{n} .
$$

Proof: Let $\alpha=\sqrt{\rho-1}-1$ so that $\alpha \geqslant-1$. Taking $c=\left(c_{1}, \ldots, c_{n}\right) \in \mathbb{C}^{n}$, we see that

$$
\begin{aligned}
\langle M c, c\rangle & =\sum_{i=1}^{n} \sum_{j=1}^{n} \frac{\bar{c}_{i} c_{j}}{\left(1-\bar{\lambda}_{j} \lambda_{i}\right)^{(\alpha+1)^{2}+1}} \\
& =\left\langle\sum_{j=1}^{n} c_{j} k_{\lambda_{j}}^{(\alpha+1)^{2}-1}, \sum_{i=1}^{n} c_{i} k_{\lambda_{i}}^{(\alpha+1)^{2}-1}\right\rangle_{L_{(\alpha+1)^{2}-1}^{2}} \\
& \geqslant 0,
\end{aligned}
$$

from which our assertion follows and

$$
\langle M c, c\rangle=\left\|\sum_{j=1}^{n} c_{j} k_{\lambda_{j}}^{(\alpha+1)^{2}-1}\right\|_{L_{(\alpha+1)^{2}-1}^{2}}^{2} .
$$

As a consequence of Lemma 1, we see that any matrix of the form

$$
\left[\frac{1}{\left(1-\overline{\varphi\left(\lambda_{j}\right)} \varphi\left(\lambda_{i}\right)\right)^{\rho}}\right]_{i, j=1}^{n},
$$

where $\varphi$ is a self-map of $D$, must also be positive semi-definite and so is

$$
\left[\frac{1}{\left(1-\left|\varphi\left(\lambda_{j}\right)\right|^{2}\right)^{\rho}}\right]_{j=1}^{n}
$$

as required.

\section{NORM INEQUALITIES}

The proof of the major theorem relies heavily on the use of Schur products. Recall that, for any two $n \times n$ matrices $A=\left[a_{i j}\right]_{i, j=1}^{n}$ and $B=\left[b_{i j}\right]_{i, j=1}^{n}$, the Schur (or Hadamard) product $A \circ B$ is defined by the following rule $A \circ B=\left[a_{i j} b_{i j}\right]_{i, j=1}^{n}$. That is, the Schur product is obtained by entrywise multiplication. A proof of the following result appears in Ref. 9.

Proposition 2 (Schur Product Theorem) If $A$ and $B$ are $n \times n$ positive semi-definite matrices, then $A \circ B$ is also positive semi-definite.

We are now in position to state the main result, a theorem that allows us to compare the norms of $C_{\varphi}$ on certain weighted spaces.

Theorem 1 Take $\beta \geqslant e_{\alpha}:=(\alpha+1)^{2}-1 \geqslant-1$ and let $\varphi$ be an analytic self-map of $D$. Then

$$
\left\|C_{\varphi}\right\|_{L_{\beta}^{2}} \leqslant\left\|C_{\varphi}\right\|_{L_{e_{\alpha}}^{2}}^{\gamma}
$$

whenever the quantity $\gamma:=(\beta+2) /\left(e_{\alpha}+2\right)$ is an integer.

Proof: Assume that $\gamma=(\beta+2) /\left(e_{\alpha}+2\right)$ is an integer. Fix a natural number $n$ and let $i, j \in\{1,2, \ldots, n\}$. A difference of higher powers factorization shows that

$$
\begin{aligned}
& \frac{\left\|C_{\varphi}\right\|_{L_{e_{\alpha}}^{2}}^{2 \gamma}}{\left.1-\overline{\lambda_{j}} \lambda_{i}\right)^{\beta+2}}-\frac{1}{\left(1-\overline{\varphi\left(\lambda_{j}\right)} \varphi\left(\lambda_{i}\right)\right)^{\beta+2}} \\
& =\left(\frac{\left\|C_{\varphi}\right\|_{L_{e_{\alpha}}^{2}}^{2}}{\left(1-\overline{\lambda_{j}} \lambda_{i}\right)^{e_{\alpha}+2}}-\frac{1}{\left(1-\overline{\varphi\left(\lambda_{j}\right)} \varphi\left(\lambda_{i}\right)\right)^{e_{\alpha}+2}}\right) \\
& \times \sum_{k=0}^{\gamma-1}\left(\frac{\left\|C_{\varphi}\right\|_{L_{e_{\alpha}}^{2}}^{2 k}}{c_{k} d_{k}}\right)
\end{aligned}
$$

where $c_{k}=\left(1-\overline{\lambda_{j}} \lambda_{i}\right)^{k\left(e_{\alpha}+2\right)}$ and $d_{k}=(1-$ $\left.\overline{\varphi\left(\lambda_{j}\right)} \varphi\left(\lambda_{i}\right)\right)^{\left(e_{\alpha}+2\right)(\gamma-k-1)}$. Then

$$
\begin{aligned}
& \frac{\left\|C_{\varphi}\right\|_{L_{e_{\alpha}}^{2}}^{2 \gamma}}{\left(1-\left|\lambda_{j}\right|^{2}\right)^{\beta+2}}-\frac{1}{\left(1-\left|\varphi\left(\lambda_{j}\right)\right|^{2}\right)^{\beta+2}} \\
& =\left(\frac{\left\|C_{\varphi}\right\|_{L_{e_{\alpha}}^{2}}^{2}}{\left(1-\left|\lambda_{j}\right|^{2}\right)^{e_{\alpha}+2}}-\frac{1}{\left(1-\left|\varphi\left(\lambda_{j}\right)\right|^{2}\right)^{e_{\alpha}+2}}\right) \\
& \quad \times \sum_{k=0}^{\gamma-1}\left(\frac{\left\|C_{\varphi}\right\|_{L_{e_{\alpha}}^{2}}^{2 k}}{a_{k} b_{k}}\right)
\end{aligned}
$$


where $a=\left(1-\left|\lambda_{j}\right|^{2}\right)^{k\left(e_{\alpha}+2\right)}$ and $b=(1-$ $\left.\left|\varphi\left(\lambda_{j}\right)\right|^{2}\right)^{\left(e_{\alpha}+2\right)(\gamma-k-1)}$.

Since the preceding equation holds for all $i$ and $j$, we obtain the following matrix equation:

$$
\begin{aligned}
& M\left(\left\|C_{\varphi}\right\|_{L_{e_{\alpha}}^{2}}^{\gamma}, n, \beta\right) \\
& =M\left(\left\|C_{\varphi}\right\|_{L_{e_{\alpha}}^{2}}, n, \alpha(\alpha+2)\right) \\
& \quad \times \sum_{k=0}^{\gamma-1}\left(\frac{\left\|C_{\varphi}\right\|_{L_{e_{\alpha}}^{2}}^{2 k}}{e_{k} f_{k}}\right)_{i, j=1}^{n}
\end{aligned}
$$

where $e_{k}=\left(1-\overline{\lambda_{j}} \lambda_{i}\right)^{k\left(e_{\alpha}+2\right)}$ and $f_{k}=(1-$ $\left.\overline{\varphi\left(\lambda_{j}\right)} \varphi\left(\lambda_{i}\right)\right)^{\left(e_{\alpha}+2\right)(\gamma-k-1)}$. This implies the matrix $M\left(\left\|C_{\varphi}\right\|_{L_{e_{\alpha}}^{2}}, n, \alpha(\alpha+2)\right)$ is positive semi-definite by Proposition 1 .

Lemma 1, together with Proposition 2, dictates that every term in the matrix sum on the right-hand side of (5) is positive semi-definite, so the sum itself is positive semi-definite. Therefore Proposition 1 shows that $M\left(\left\|C_{\varphi}\right\|_{L_{e_{\alpha}}^{2}}^{\gamma}, n, \beta\right)$ must also be positive semidefinite.

Since this assertion holds for every natural number $n$, we obtain by Proposition 1 that $\left\|C_{\varphi}\right\|_{L_{\beta}^{2}} \leqslant$ $\left\|C_{\varphi}\right\|_{L_{e_{\alpha}}^{2}}^{\gamma}$.

Taking $\alpha=0, \alpha=-1$ and $\alpha=-2$, we obtain ${ }^{8}$ the following corollaries.

Corollary 1 Let $\varphi$ be an analytic self-map of D. Then

$$
\left\|C_{\varphi}\right\|_{L_{\beta}^{2}} \leqslant\left\|C_{\varphi}\right\|_{H^{2}}^{\beta+2}
$$

whenever $\beta$ is a non-negative integer. In particular, $\left\|C_{\varphi}\right\|_{L^{2}} \leqslant\left\|C_{\varphi}\right\|_{H^{2}}^{2}$.

Corollary 2 Let $\varphi$ be an analytic self-map of D. Then

$$
\left\|C_{\varphi}\right\|_{L_{\beta}^{2}} \leqslant\left\|C_{\varphi}\right\|_{L^{2}}^{(\beta+2) / 2}
$$

whenever $\beta$ is a positive even integer.

Theorem 2 Take $\beta \geqslant e_{\alpha}:=(\alpha+1)^{2}-1 \geqslant-1$ and let $\varphi$ be an analytic self-map of D. Suppose that $\gamma=$ $(\beta+2) /\left(e_{\alpha}+2\right)$ is an integer. If $C_{\varphi}$ is cosubnormal on $L_{e_{\alpha}}^{2}$, then it is also cosubnormal on $L_{\beta}^{2}$.

Cowen ${ }^{10}$ only stated this result for $\alpha=-1$, but an identical argument works for $\alpha>-1$. The proof makes use of Proposition 1 in a similar fashion to that of Theorem $1^{8}$.

\section{REFERENCES}

1. Cowen C, MacCluer B (1995) Composition Operators on Spaces of Analytic Functions, CRC Press, Boca Raton, FL.

2. Basor E, Retsek D (2006) Extremal non-compactness of composition operators with linear fractional symbol. J Math Anal Appl 322, 749-63.

3. Bourdon P, Fry E, Hammond C, Spofford C (2004) Norms of linearfractional composition operators. Trans Am Math Soc 356, 2459-80.

4. Hammond C (2003) On the norm of a composition operator with linear fractional symbol. Acta Sci Math 69, 813-29.

5. Hammond C (2006) Zeros of hypergeometric functions and the norm of a composition operator. Comput Meth Funct Theor 6, 37-50.

6. Richman A (2003) Subnormality and composition operators on the Bergman space. Integr Equat Operat Theor 45, 105-24.

7. Carswell B, Hammond C (2006) Composition operators with maximal norm on weighted Bergman spaces. Proc Am Math Soc 134, 2599-605.

8. Hammond C, Patton LJ (2010) Norm inequalities for composition operators on Hardy and weighted Bergman spaces. In: Ball JA, et al (eds) Operator Theory: Advances and Applications, vol. 202, pp 265-72.

9. Horn R, Johnson C (1985) Matrix Analysis, Cambridge Univ Press, Cambridge.

10. Cowen C (1992) Transferring subnormality of adjoint composition operators. Integr Equat Operat Theor 15, 167-71. 